\section{REAL AND APPARENT SOURCES OF POLYDISPERSITY IN MOLECULAR WEIGHT DISTRIBUTIONS FROM RADICAL POLYMERIZATION}

\author{
Gregory T. Russell
}

\author{
Department of Chemistry \\ University of Canterbury \\ Private Bag 4800 \\ Christchurch, 8140 \\ New Zealand \\ greg.russell@canterbury.ac.nz
}

\section{Introduction}

A Holy Grail of the macromolecular chemist is to achieve the 'purity' in size of micromolecular chemistry. In a real sense this quest is like that of Sisyphus in Greek mythology, who was eternally condemned to rolling a boulder uphill. The macromolecular chemist will also never reach the top of the hill, because the statistical nature of all polymerizations makes true monodispersity in size impossible to achieve by chemical means. Be that as it may, the advent of living radical polymerization (LRP) has reawakened the dream, and over the last decade there have been literally tens of thousands of papers in which workers have used this tremendous new chemical principle to strive towards the magical polydispersity index of 1 . Given this it is timely to take stock of recent investigations into factors that influence the polydispersity of molecular weight distributions from radical polymerization.

\section{Background Revision}

Classical Radical Polymerization. It has long been known that for steady-state radical polymerization with chain-length-independent reactivities, the instantaneous polydispersity index, $P D I$, must lie between the limits of 1.5 and 2, where the lower limit is for the case of all dead-chain formation by combination, while the upper limit is for all dead-chain formation by disproportionation and/or transfer.

Ideal Living Polymerization. This yields a Poisson distribution of chain lengths, for which $P D I=1+\left(D P_{\mathrm{n}}-1\right)^{-1}$, where $D P_{\mathrm{n}}$ is the numberaverage degree of polymerization. Figure 1 shows these distributions for $D P_{\mathrm{n}}$ $=51$ and 101. Two important points may be made: (1) Even though PDI is very close to 1 , in terms of micromolecular idylls there is still a lot of heterogeneity in size. Yet this is the very best one can do with polymerization in terms of monodispersity. This emphasizes the point above that the stochastic nature of polymerization makes unreachable the Holy Grail of perfect monodispersity: it is simply not possible to make all growing chains march in unison. (2) Even though $P D I$ is smaller for the case of $D P_{\mathrm{n}}=101$, this distribution has a greater absolute broadness. This emphasizes that $P D I$ is only a measure of relative broadness.

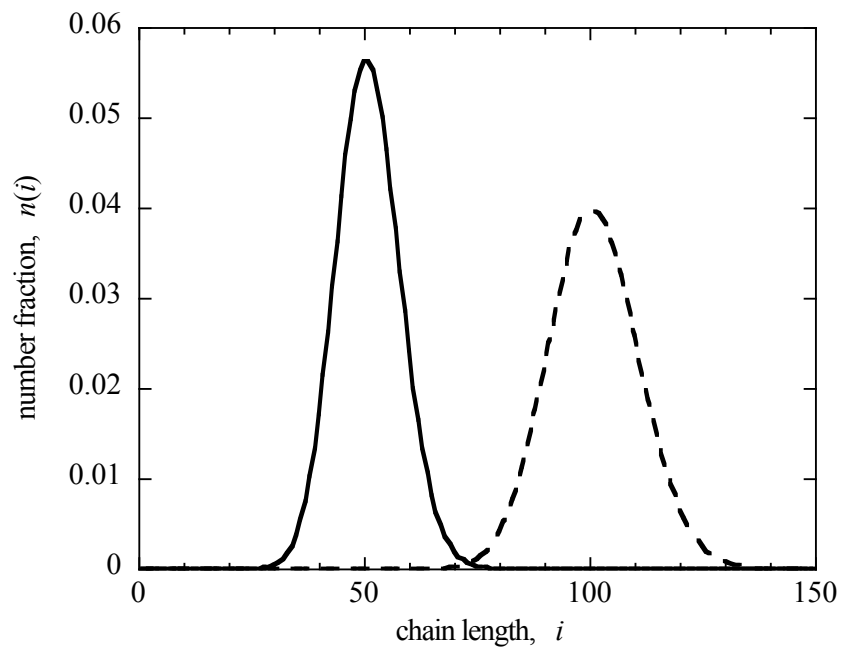

Figure 1. Poisson chain-length distribution for $D P_{\mathrm{n}}=51($ left; $P D I=1.02)$ and $D P_{\mathrm{n}}=101$ (right; $\left.P D I=1.01\right)$.

\section{Apparent Sources of Polydispersity}

SEC Broadening. Even if one succeeds in synthesizing polymer as monodisperse as in Figure 1, this will not be observed via size exclusion chromatography (SEC) because of column broadening. This is the unavoidable phenomenon of molecules of identical hydrodynamic volume having a distribution of elution times. Thus for any polymer sample the apparent molecular weight distribution (MWD) delivered by SEC is always broader than the true MWD of the sample. There is no routine way of deconvoluting the apparent MWD to obtain the true MWD. Therefore we have used an algorithm of Lämmel et al. ${ }^{1}$ to develop a reverse-engineering approach in which calculated MWDs are broadened by simulation until exact agreement with (apparent) experimental MWDs is obtained. ${ }^{2}$ This provides a relatively simply way of unraveling the effect of SEC broadening from (apparent) experimental MWDs.

SEC Calibration. Because only a limited variety of polymers are available as SEC standards, in carrying out SEC it is common practice to use standards that are different to the polymer of interest. This has recently been investigated by Guillaneuf and Castignolles. ${ }^{3}$ They have shown that only when the two polymers have the same $a$ (Mark-Houwink-Kuhn-Sakurada exponent) does SEC return the correct PDI. However, unlike with SEC broadening, this effect may go either way: if $a$ (sample) $<a$ (standard), then the apparent $P D I$ is less than the true $P D I$ of the sample, while if $a$ (sample) $>$ $a$ (standard), then the sample will appear broader than it really is. There seems little doubt that this phenomenon must routinely contribute to misinterpretation of experimental results, especially in the field of LRP.

\section{Real Sources of Polydispersity}

Chain-Length-Dependent Termination. The classical theory of radical polymerization assumes chain-length-independent reactvities. It is now accepted that this is inappropriate for termination. The simplest model for chain-length-dependent termination (CLDT) that also has a physical basis is the following power law:

$$
k_{\mathrm{t}}^{i, i}=k_{\mathrm{t}}^{1,1} i^{-\alpha}
$$

Here $k_{\mathrm{t}}^{i, i}$ is the rate coefficient for termination between radicals of chain length $i$, while $\alpha$ is a parameter quantifying the strength of the chain-length dependence. In regrettably ignored work, Olaj et al. ${ }^{4}$ have shown that eq 1 leads to

$$
n_{\mathrm{disp}}(i)=C i^{-\alpha / 2} \exp \left(-C i^{p}\right)
$$

as the (normalized) chain-length distribution (CLD) for disproportionation, and

$$
n_{\text {comb }}(i)=A^{2} p i^{1-\alpha} \exp \left(-A i^{p}\right)
$$

as the (normalized) CLD for combination, where $C=\left(2 R_{\mathrm{i}} k_{\mathrm{t}}^{1,1}\right)^{0.5} /\left(k_{\mathrm{p}} C_{\mathrm{M}}\right)$ is the inverse of the classical kinetic chain length, $p=1-\alpha / 2, C^{`}=C / p$ and $A=$ $4 C^{\%} /(4-\alpha)$. Evaluations of eq 2 for $\alpha=0$ and $\alpha=0.3$, with $C$ varied so as to give the same $D P_{\mathrm{n}}$, are shown in Figure 2.

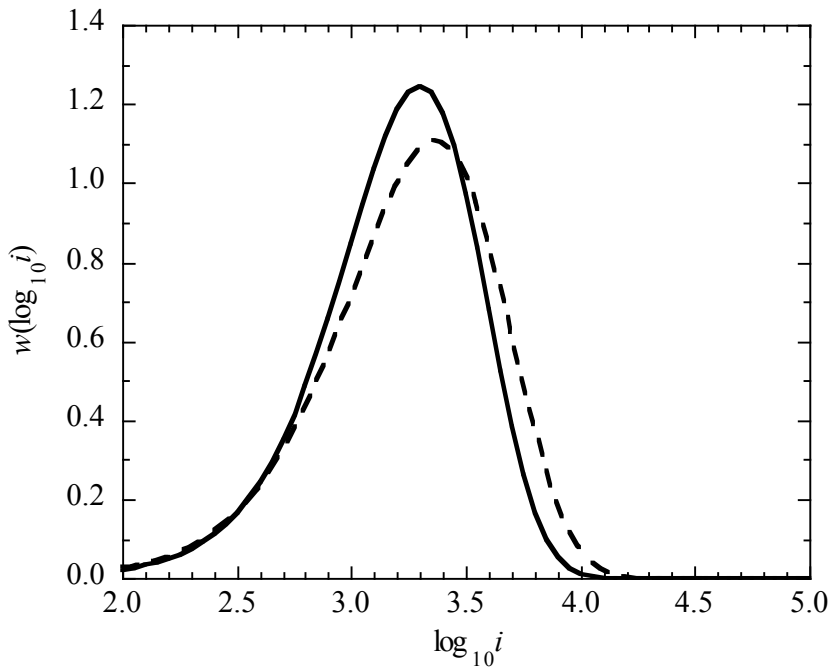

Figure 2. Normalized weight fraction, $w$, as a function of chain length, $i$, for $\alpha=0$ (full line; chain-length-independent termination) and $\alpha=0.3$ (dashed line; CLDT), where dead-chain formation is by disproportionation. For both distributions $D P_{\mathrm{n}}=1000$. 
The broadening effect of CLDT on MWDs is evident in Figure 2. The same also holds for combination, as shown in Figure 3. Incidentally, both figures show that $D P_{\mathrm{n}}$ is a poor gauge of the central position of SEC distributions.

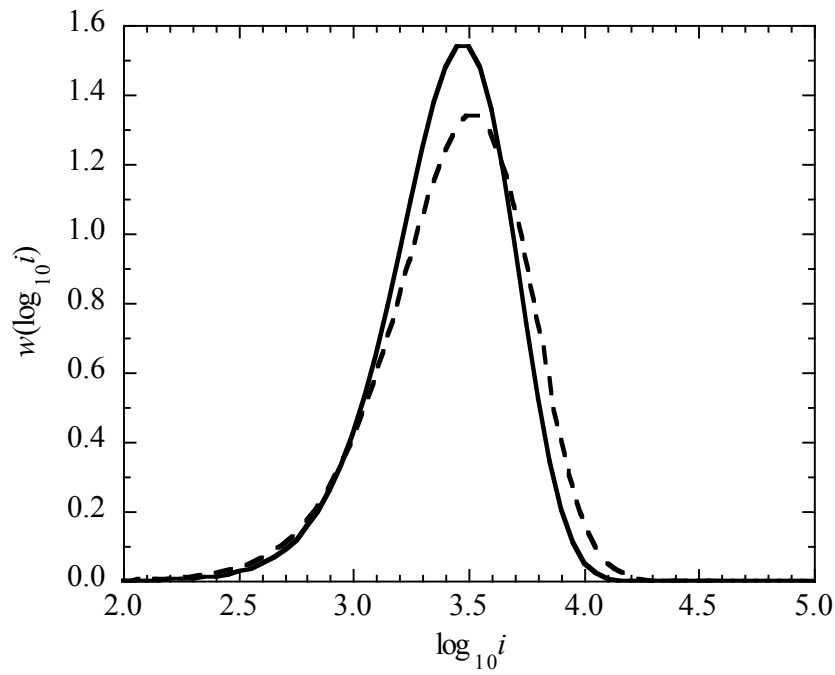

Figure 3. Normalized weight fraction, $w$, as a function of chain length, $i$, for $\alpha=0$ (full line; chain-length-independent termination) and $\alpha=0.3$ (dashed line; CLDT), where dead-chain formation is by combination. For both distributions $D P_{\mathrm{n}}=2000$.

Where both disproportionation and combination are operative, one should use

$$
n_{\text {mixed }}(i)=F_{\mathrm{n}} n_{\text {disp }}(i)+\left(1-F_{\mathrm{n}}\right) n_{\text {comb }}(i)
$$

Here $F_{\mathrm{n}}=2 \lambda(1+\lambda), \lambda$ being the fraction of termination events that take place by disproportionation.

Using the above equations one may show that ${ }^{4,5}$

$$
P D I=\frac{1+\lambda}{2}\left\{\frac{\Gamma\left(\frac{6-\alpha}{2-\alpha}\right)}{\left[\Gamma\left(\frac{4-\alpha}{2-\alpha}\right)\right]^{2}}+1-\lambda\right\}
$$

Equation 5 is evaluated in Figure 4, which quantifies the broadening of CLDs that is displayed in Figures 2 and 3. It is evident that as CLDT becomes stronger, MWDs become broader and broader.

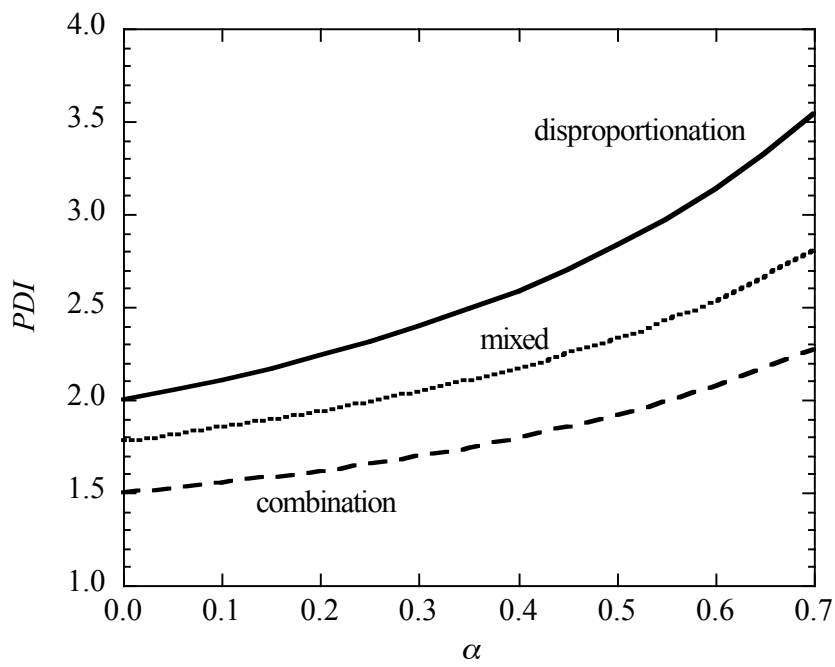

Figure 4. Polydispersity index as a function of CLDT exponent $\alpha$ for disproportionation, combination and $\lambda=0.333$ ("mixed"), as indicated.
Chain-Length-Dependent Propagation. For $D P_{\mathrm{n}}$ of order 100 or less, radical polymerization kinetics are affected by chain-length-dependent propagation (CLDP). This phenomenon results in very small radicals growing relatively quickly, and thus - opposite to CLDT - there is narrowing of the MWD. ${ }^{6}$ The effect can be substantial, with PDI of 1.6 having been observed for transfer-controlled systems, ${ }^{7}$ as opposed to the classical kinetics value of 2 for such systems. Further, these experimental results have been well reproduced by modeling that incorporated CLDP. ${ }^{7,8}$

\section{Living Radical Polymerization}

All the effects above are relevant to the more complicated process of LRP. Most obviously, the instrumental effects apply regardless of how polymer is synthesized. As for CLDP and CLDT, while they are (hopefully) only background processes, they are nevertheless responsible for the broad base of the MWD, and in fact this base can be largely what determines the deviation of the $P D I$ from the ideal value of $1+\left(D P_{\mathrm{n}}-1\right)^{-1}$.

Where termination is concerned, it is possible to regard LRP as an analogue of single-pulse pulsed-laser polymerization (SP-PLP). ${ }^{9}$ Taking this view, then the 'background' portion of the MWD from LRP should be like that from SP-PLP, ${ }^{10}$ onto which a Poisson distribution, as in Figure 1, is superimposed as a result of the LRP process. It may be possible to explore these notions in order to obtain a relatively simple description of (overall) PDI for LRP.

\section{Conclusion}

Many factors contribute to the broadness of molecular weight distributions in radical polymerization. One should beware simple descriptions that seek to ascribe $P D I$ totally to one factor; life is rarely (if ever) that straightforward.

Acknowledgements. Greg Smith, Kim van Berkel, Hans Heuts, Chris Barner-Kowollik and Bob Gilbert are all acknowledged for their contributions, great and small, to the author's publications associated with this work.

References

(1) Buback, M.; Busch, M.; Lämmel, R. A. Macromol. Theory Simul., 1996, 5,845 .

(2) van Berkel, K. Y.; Russell, G. T.; Gilbert, R. G. Macromolecules, 2005 38,3214 .

(3) Guillaneuf, Y.; Castignolles, P. J. Polym. Sci., Polym. Chem. Ed., 2008, $46,897$.

(4) Olaj, O. F.; Zifferer, G.; Gleixner, G. Macromolecules, 1987, 20, 839.

(5) Barner-Kowollik, C.; Russell, G. T. Prog. Polym. Sci. 2008, submitted.

(6) Heuts, J. P. A.; Russell, G. T.; Smith, G. B. Aust. J. Chem., 2007, 60, 754.

(7) Smith, G. B.; Russell, G. T.; Yin, M.; Heuts, J. P. A. Eur. Polym. J., 2005, 41, 225

(8) Smith, G. B.; Heuts, J. P. A.; Russell, G. T. Macromol. Symp., 2005, 226,133 .

(9) Barner-Kowollik, C.; Buback, M.; Egorov, M.; Fukuda, T.; Goto, A.; Olaj, O. F.; Russell, G. T.; Vana, P.; Yamada, B.; Zetterlund, P. B. Prog. Polym. Sci., 2005, 30, 605.

(10) Smith, G. B.; Russell, G. T. Z. Phys. Chem. (Munich), 2005, 219, 295. 